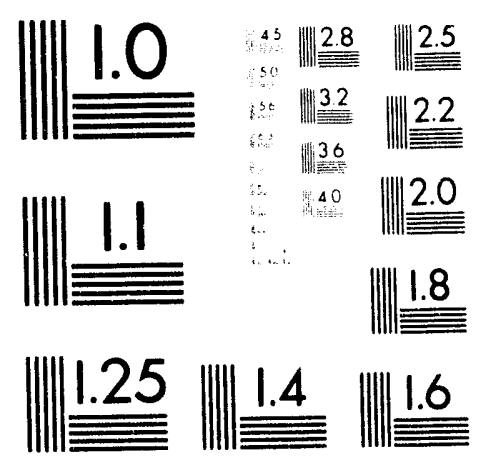



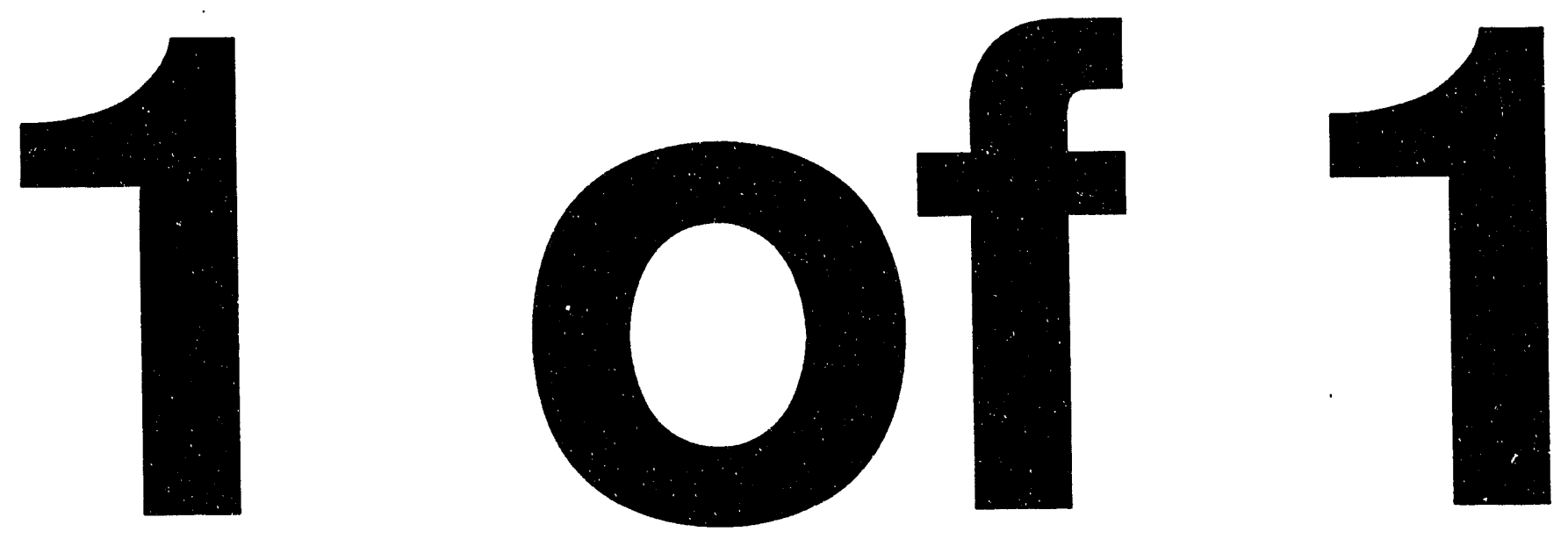


\title{
Computer Studies of a Combined-Function Bend Magnet for a Proposed Redesign of the SLAC SLC Damping Rings*
}

\author{
R. A. Early and T. O. Raubenheimer
}

Stanford Linear Accelerator Center, Stanford University, Stanford, CA 94309

\section{Abstract}

A proposed redesign of the SLAC SLC Damping Ring requires a combined-function bending magnet. The magnet will operate with a main field of $1.8338 \mathrm{~T}$, and quadrupole and sextupole gradients $d B_{y} / d x, d^{2} B_{y} / d x^{2}$ of $-14.1 \mathrm{~T} / \mathrm{m}$ and $-477 \mathrm{~T} / \mathrm{m}^{2}$, respectively. Because the orbit sagitta in the magnet is in excess of $2 \mathrm{~cm}$, the pole will be curved with a $2 \mathrm{~m}$ radius of curvature. Furthermore, since the current must be variable over a range of \pm 2 percent, we have considered using vanadium permendur poles to avoid adverse saturation effects. Studies were done using POISSON in 2-D and TOSCA for 3-D end effects.

\section{INTRODUCTION}

A possible redesign of the Sta ford Linear Collider (SLC) damping rings is under consideration [1]. The new ring is designed to have horizontal equilibrium emittances of $\gamma \epsilon_{x}=9 \times 10^{-6} \mathrm{~m}$-rad; this is over three times smaller than that in the current SLC damping rings. The reduction in the emittance is achieved by replacing the present bending magnets with combined function magnets. The bends are $69 \mathrm{~cm}$ long and operate with a main field of $1.83 \mathrm{~T}$ for a beam energy of $1.19 \mathrm{GeV}$. They have both quadrupole and sextupole gradients of $d B_{y} / d x=-14.1$ $\mathrm{T} / \mathrm{m}$ and $d^{2} B_{\mathrm{y}} / d x^{2}=-477 \mathrm{~T} / \mathrm{m}^{2}$. Each magnet will replace two of the present SLC damping-ring bend magnets which are $30.4 \mathrm{~cm}$ long and have peak fields of $2.02 \mathrm{~T}$. Finally, the beam orbit has a 2.2 meter radius of curvature and a sagitta of $2.7 \mathrm{~cm}$ in the $68-\mathrm{cm}$ bends. Thus, the pole needs to be curved, following the beam trajectory, to prevent variation of the bending field and gradient through the magnet.

The magnetic gap in the bends was chosen to be equal to the aperture in the quadrupoles, $1.3 \mathrm{~cm}$ radius. This allows a constant radius vacuum chamber without the aperture transitions that can increase the impedance of the ring. But, this 2.6- $\mathrm{cm}$ gap is $30 \%$ larger than the gap in the current damping ring bends, and thus makes it harder to achieve the desired field quality.

We decided wo design the pole with vanadium permendur because of its high saturation inductance. This allows us to achieve the desired field quality while reducing the fringe fields. It also prevents differential saturation across the pole so that when the main field is changed by $\pm 2 \%$, the relative strength of the gradients changes by less than $10^{-3}$ and higher-order multipoles are not introduced; this would allow the energy of the damping ring to be varied in a straightforward manner.

* Work supported by Department of Energy, contract DE-AC03-76SF00515.

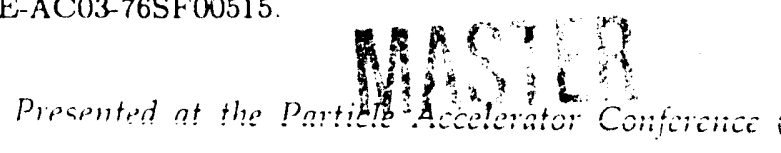

In the following sections, we describe the design calculations using POISSON [2] and TOSCA [3], then we discuss some of the properties of and experience with vanadium permendur, and finally, we describe the construction tolerance calculations.

\section{DESIGN CALCULATIONS}

The bending magnets are $18^{\circ}$ sector-bend magnets. The beam enters and exits at right angles to the pole face, although we plan to introduce a rotation to compensate for quadrupole roll-off due to end effects. The first computer studies were done using POISSON, starting from an approximation to the poleface that was obtained with potential theory. The poleface optimization program MIRT [4] was then used to shape the pole such that the required field harmonics were obtained within a $1-\mathrm{cm}$ radius of the beam path. The pole profile is shown in Figure 1. The pole is $11.4-\mathrm{cm}$ wide at the base and has a height of 7.3 $\mathrm{cm}$. It is curved with a 2.2 -meter radius of curvature and the beam path is offset $1.6 \mathrm{~cm}$ horizontally from the center of the pole where the half gap is $1.3 \mathrm{~cm}$.

A profile of the full magnet is shown in Figure 2. The yoke is only slightly larger than those in the existing SLC damping-ring bend magnets. An extra layer of coil has been used so that the 1.8 Tesla field could be generated with 300 amps; this is well within the capacity of the present power supplies.

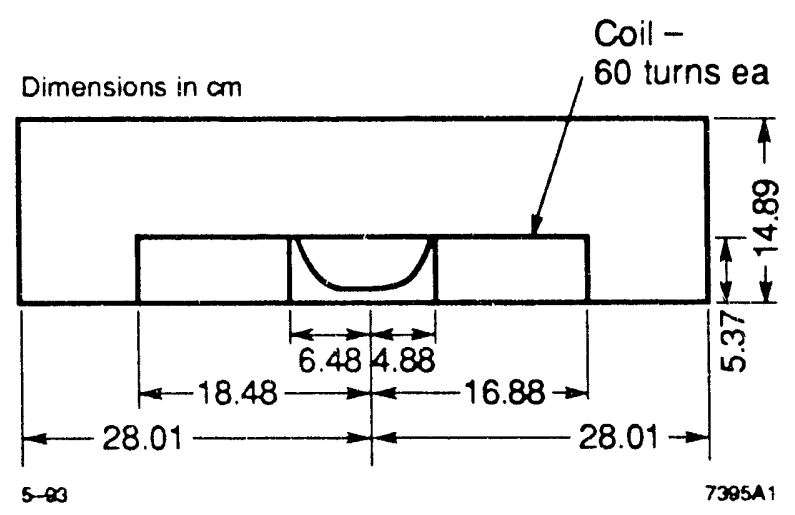

Figure 1 Pole profile of the bending magnet; the main magnetic field is $1.83 \mathrm{~T}$ with gradients of $u B_{y} / d x=-14.1 \mathrm{~T} / \mathrm{m}$ and $d^{2} B_{v} / d x^{2}=-477 \mathrm{~T} / \mathrm{m}^{2}$.

TOSCA was used to study the effects of the ends on harmonic content. Two views of the mesh are shown in Figures 3 and 4 . The coil is a saddle coil because there is very little space between magnets in the damping rings. We used the version of TOSCA that is running on the NERSC CRAY 2 at Lawrence Livermore National Laboratory. The curvature of the magnet was ignored in the

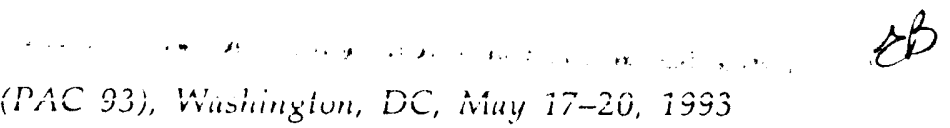




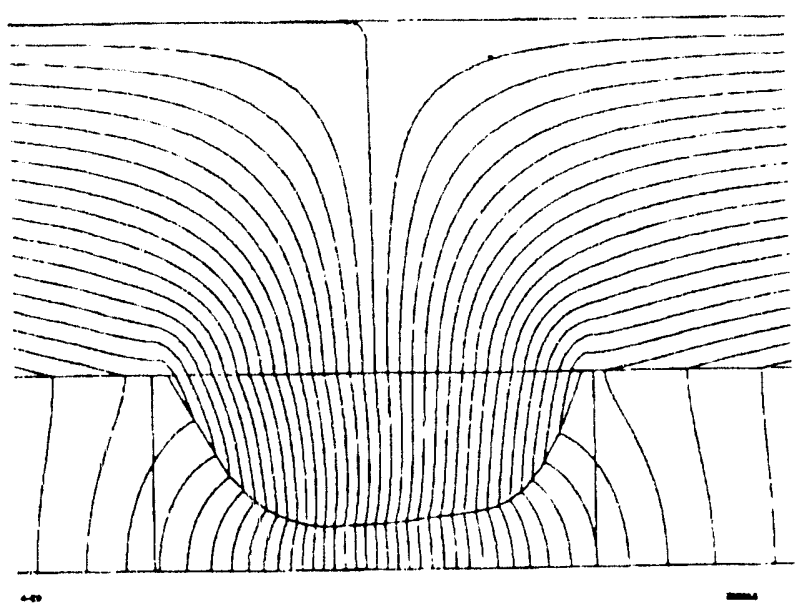

Figure 2 Profile of the bending magnet; the pole is designed with Vanadium Permendur while the yoke is to be constructed of magnet iron; all dimensions are in centimeters.

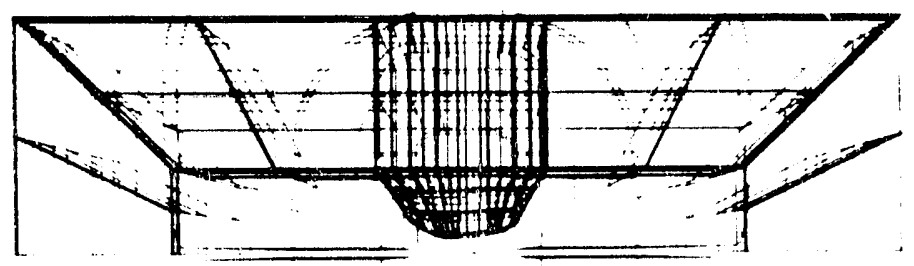

Figure 3 TOSCA finite element mesh for the combined function bending magnet showing the coil position. Only one half of the iron need be modeled, but the full set of coils is required.

3-D modeling. The problem was run with cylindrical symmetry in POISSON with a $2.2-\mathrm{m}$ radius and no significant change was observed in field distribution.

The harmonic content versus longitudinal position in the magnet is shown in Figure 5. Field uniformity within the magnet is good and the fringing fields decay rapidly. Without chamfered nose pieces, the effective length, obtained from TOSCA, is $6 \%$ longer than the physical iength. The two-dimensional harmonics obtained with TOSCA varied from MIRT results because the number of points on the poleface was smaller. The quadrupole component

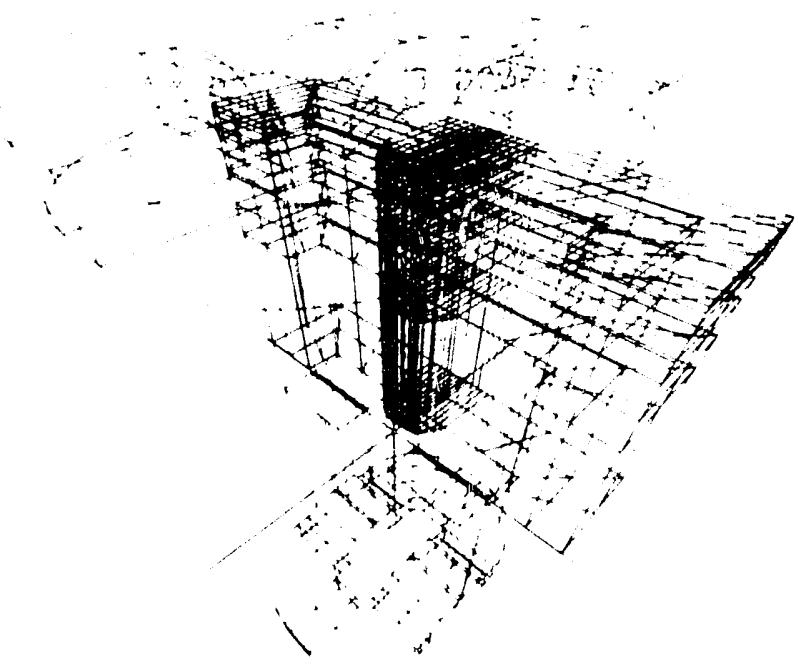

Figure 4 Alternate view of the TOSCA finite element mesh for the bending magnet.

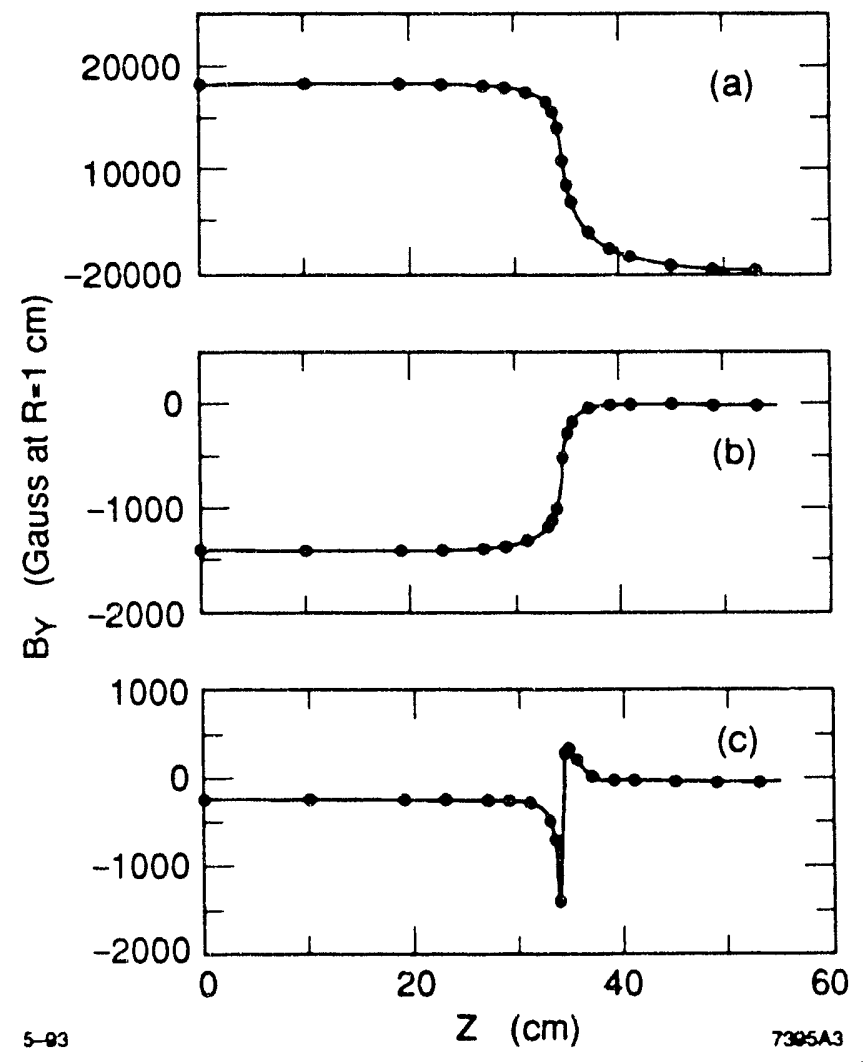

Figure 5 Harmonic content versus longitudinal position in the bend; (a) main field, (b) quadrupole component at $r=1 \mathrm{~cm}$, and (c) sextupole component at $\mathrm{r}=1 \mathrm{~cm}$.

was 0.36 percent higher and the sextupole 5.2 percent lower. When integrated over the length of the magnet, the quadrupole component decreases in absolute value by 6 percent, while the sextupole increases in absolute value by 8 percent. Work is in progress to correct these effects with a combination of pole-end rotation and shaping. 


\section{VANADIUM PERMENDUR}

Vanadium permendur is an alloy of iron and cobalt in equal proportions with about 2 percent vanadium and has been discussed in previous papers $[5,6]$. It has a steep $B H$ curve and a high saturation induction $\left(B-\mu_{0} H\right)$ near 2.3 $\mathrm{T}$, that makes it useful in the design of magnets in cases where fields are high, but constant magnetic properties are desired.

So far, five magnets using vanadium permendur poles have been built at SLAC. We changed our annealing procedure to a lower temperature than used in Reference 5. Machining can be difficult unless the material is annealed first. At present, our procedure calls for a four-hour anneal at $750^{\circ} \mathrm{C}$ with a slow cool. A second annealing process is done before the final machining. The procedure leaves roughly $0.5 \mathrm{~mm}$ for final machining. One problem which was encountered with our first magnet of this type was that the material had been forged and some distortion was encountered during annealing. This is the reason for doing a sccond anneal before final machining.

\section{CONSTRUCTION ERRORS}

It was initially thought that the construction tolerances on this combined-function magnet would be very tight. Fortunately, this does not seem to be the case. Dynamic aperture calculations show that raridorn quadrupole and sextupole field tolerances of $\Delta B_{1} \lesssim 0.14 \mathrm{~T} / \mathrm{m}$ and $\Delta B_{2} \lesssim 9.5 \mathrm{~T} / \mathrm{m}^{2}$, and random and systematic octupole field tolerances of $\Delta B_{3} \leqslant 50 \mathrm{~T} / \mathrm{m}^{3}$ are acceptable; higher multipoles were not considered. In addition, tuning studies show that recovery is possible from even larger systematic quadrupole and sextupole field errors. Finally, the emittance coupling sets a tolerance of $\Delta S_{1} \lesssim 0.1 \mathrm{~T} / \mathrm{m}$ on the skew quadrupole field

Thus, we have studied five sources of error: accuracy of the alignment fiducials relative to the magnetic center, relative horizontal alignment of the two poles, variation of the magnet gap, fatness of the pole base and the yoke, and the accuracy of the pole contour. The first three sources do not need explanation. The fourth effect, the flatness of the pole base and the yoke, principally changes the quadrupole component and skew quad component because the angle of the pole contour is changed when the pole is bolted to the yoke.

The last effect refers to the accuracy of the pole contour. Assuming a systematic sinusoidal variation of the pole contour: $f(x)=f_{\text {design }}(x)+a \sin (k x+\phi)$, we can calculate the multipole component using a perturbative expansion:

$$
\left|\frac{\Delta B_{n}}{B_{0}}\right|=a \frac{k^{n+1}}{\sinh (k h)},
$$

where $h$ is the half gap and $k$ is $2 \pi / \lambda$ of the error. The tolerance listed in Table 1 corresponds to the sextupole tolerance; the quadrupole tolerance is a factor of three looser and the octupole tolerance will be tighter. Although this tolerance is tight, it is important to note that it applies only to a systematic error along the entire length of the pole. If the phase of the sinusoidal error varies along the magnet, the effect will average to a smaller contribution. Finally, these results were verified with POISSON calculations.

Table 1. Construction tolerances.

\begin{tabular}{|c|c|}
\hline Fiducial: $X$ & $\pm 150 \mu \mathrm{m}$ \\
\hline Fiducial: $Y$ & $\pm 100 \mu \mathrm{m}$ \\
\hline Pole offset: $x$ & $\pm 300 \mu \mathrm{m}$ \\
\hline Full gap: $g$ & $\pm 140 \mu \mathrm{m}$ \\
\hline Flatness: $y$ & $\pm 50 \mu \mathrm{m}$ \\
\hline Pole contour: $a$ with $\lambda=2.5 \mathrm{~cm}$ & $5 \mu \mathrm{m}$ \\
\hline
\end{tabular}

\section{DISCUSSION}

We described a high-field combined-function bending magnet that uses vinadium permendur for the magnet pole. We conclude that it should be feasible to build the magnet, provided that proper quality control is exercised during construction. Previous experience has shown that vanadium permendur can be used in this application, although it is quite expensive at this time. We plan to measure the field quality by means of a Hall probe mounted on a computer-controlled positioning device. This device has been used to obtain field maps of several magnets at SLAC.

\section{ACKNOWLEDGEMENTS}

The POISSON/MIRT program package was run on both the SLAC IBM 9021 and the NERSC CRAY 2. TOSCA was run on the NERSC CRAY 2 and we are grateful to A. Paul for his support.

[1] T. O. Raubenheimer et. al., "A Possible Redesign of the SLAC SLC Damping Rings," presented at this conference.

[2] K. Halbach, "POISSON Lectures at LBL," unpublished (1972).

3] J. Simkin, C. W. Trowbridge, "Three-dimensional Nonlinear Electromagnetic Field Calculations, Using Scalar Potentials," IEEE Proceedings, vol 27, Pt B, Nn. 6, Nov, 1980 .

[4] K. Halbach, "A Program for Inversion of Systems Analysis and its Application to the Design of Magnets," UCRL-17436 (1967).

[5] R. Early, et. al., "Design Calculations and Measurements of a Dipole Magnet with Permendur Pole Pieces," SL.AC-PUB-4883, Proc. of 1989 IEEE Part. Acc. Conf., Chicago, IL (1989).

[6] J. Coob and R. Early, "Some Initial Results from the SLAC Permeameter," SLAC-PUB-5168, Proc of Acc. Computer Code Mig., Los Alamos, NM (1990). 

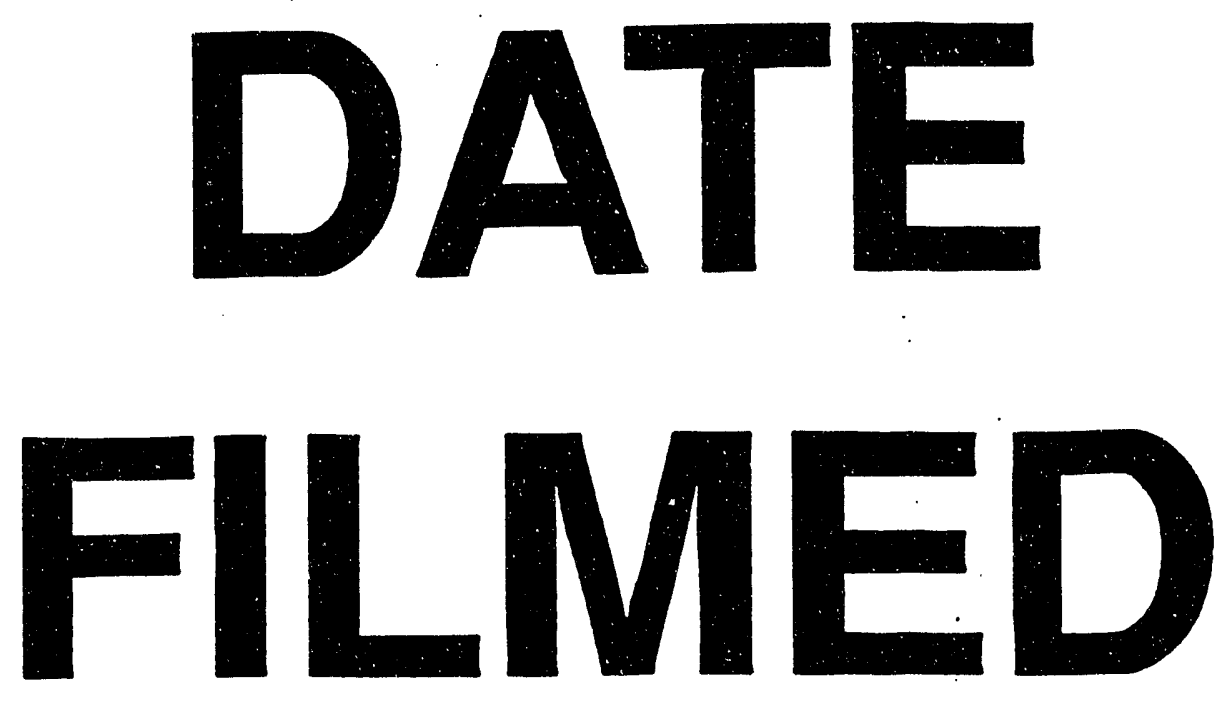

$11 / 9 / 93$
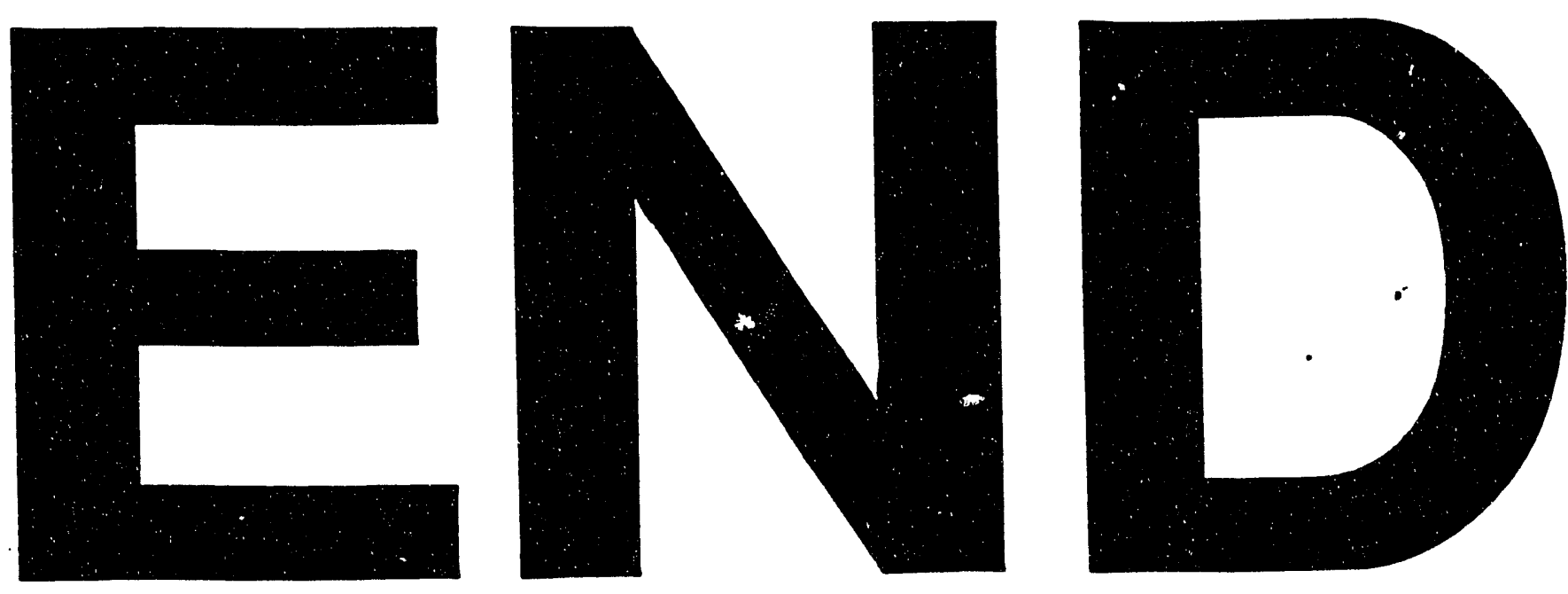
